\title{
Evolutionary games with random number of interacting players applied to access control
}

\author{
Hamidou Tembine *, Eitan Altman ${ }^{\dagger}$, Rachid El-Azouzi *, Yezekael Hayel* \\ * LIA/CERI, Univesité d'Avignon, Agroparc BP 1228, 84911 Avignon Cedex 9, France \\ $\dagger$ INRIA, Centre Sophia-Antipolis, 2004 Route des Lucioles, 06902 Sophia-Antipolis Cedex, France
}

\begin{abstract}
We study access games within a large population of mobiles that interfere with each other through many local interactions. Each local interaction involves a random number of mobiles. The games are not necessarily reciprocal as the set of mobiles causing interference to a given mobile may differ from the set of those suffering from its interference. We model and study this using the theory of evolutionary games which we extend to cover a random number of players.
\end{abstract}

Index Terms-multiple access game, evolutionary games, node distribution.

\section{INTRODUCTION}

The evolutionary game framework models competition among large populations through many local interactions, each involving a small number of users. It introduces the concept of Evolutionary Stable Strategy (ESS), as well as the population dynamics that result from the interactions between the populations. The ESS, first defined in [4], is characterized by a property of robustness against invaders (mutations). More specifically, (i) if an ESS is reached, then the proportions of each population do not change in time (ii) at ESS, the populations are immune from being invaded by other small populations. The ESS equilibrium concept is better adapted to large populations of players as it describes robustness against deviations of a whole fraction of the population as opposed to the Nash equilibrium concept that does not apply to deviations of more than a single player. We refer the reader to [11], [2] for more details on ESS and evolutionary game dynamics.

Several previous papers have already studied evolutionary games with pairwise local interactions in the context of wireless networks. Bonneau et al. have introduced evolutionary games in the context of unslotted ALOHA in [1]. They have identified conditions for the existence of non trivial ESS and have computed them explicitly. In [5], the authors considered the multiple access game and

This work was partially supported by the ANR WINEM no 06TCOM05. studied delay effect under various models of evolutionary game dynamics with asymmetric delay based on the theoretic results on stability obtained in [6]. In [7], the authors extended this model by including a regret cost, incurred when no user transmits, and studied the impact of that cost on the proportion of mobiles that transmit at equilibrium. In the last three papers, the delay is shown to have negative impact on the stability of the system.

In this paper, we extend the evolutionary game framework to allow an arbitrary, possibly random, number of players that are involved in a local interaction; we apply this to the model of [7] which we extend to more than two interacting nodes. In the context of Medium Access Game, we study the impact of the node distribution in the game area on the equilibrium stable strategies of the evolutionary game. The interaction between more than two individuals in a population is a new concept in evolutionary game theory and has a lot of application in multiple access game in wireless networks. Considering this kind of games, we use the notion of expected utility as this game is not symmetric, indeed the number of players with which a given one interacts may vary from one to another; and also non-reciprocity property. We consider the following parameters in the multiple access game: transmission cost, collision cost and regret cost. Finally, we analyze the impact of these parameters on the probability of successful transmission and give some optimization issues.

The paper is structured as follows. We first provide in the next section an evolutionary game model with random number of opponent. We then study in section III a generalized multiple access game in the context of random number of players. We compute the expression of the ESS in this typical context. After that, we analyze in section IV the probability of success transmission. Numerical solutions of replicator dynamics are investigated in section $\mathrm{V}$. 


\section{EVOLUTIONARY GAMES WITH LOCAL} INTERACTION AMONG RANDOM NUMBER OF PLAYERS

The classical evolutionary game formalism is a central mathematical tool developed by biologists for predicting population dynamics in the context of interaction between populations. In order to make use of the wealth of tools and theory developed in the biology literature, many works in the area of computer networks [6] ignore cases where local interactions between populations involve more than two individuals. This restriction limits the modeling power of evolutionary games which are not useful in a network operating at heavy load, such as adhoc networks with high density (see section IV). This motivated us in this paper to consider a random number of users interacting locally.

Consider a large population of players. Each individual needs occasionally to take some action. When doing so, it interacts with the actions of some $M$ (possibly random number of) other individuals.

\section{A. Symmetry and Reciprocity}

We shall consider throughout the paper a symmetric game in the sense that any individual faces the same type of game. All players have the same actions available, and same expected utility. We note however that the actual realizations need not be symmetric. In particular, (i) the number of players with which a given player interacts may vary from one player to another. (ii) We do not even need the reciprocity property: if player $\mathrm{A}$ interacts with player B, we do not require the converse to hold. We provide some examples of multiple access games to illustrate this non-reciprocity.

For example, we consider local interactions between transmitters; for each transmitter there corresponds a receiver. We shall say that a transmitter $\mathrm{A}$ is subject to an interaction (interference) from transmitter $B$ if the transmission from $\mathrm{B}$ overlaps that from $\mathrm{A}$, and provided that the receiver of the transmission from $A$ is within interference range of transmitter $B$.

Example 1 Consider the example depicted at Figure 1. It contains 4 sources (circles) and 3 destinations (squares). A transmission of a source $i$ within a distance $r$ of the receiver $R$, causes interference to a transmission from a source $j \neq i$ to receiver $R$. We see that Source $A$ and Source $C$ cause no interference to any other transmission but the transmission from A suffers from interference from source $\mathrm{B}$, and the one from $\mathrm{C}$ suffers from the transmission of the top most source (called D). Source B and D interfere with each other at their common destination. Thus each of the four sources suffers interference from a single other source, but except for nodes $\mathrm{B}$ and $\mathrm{D}$, the interference is not reciprocal.

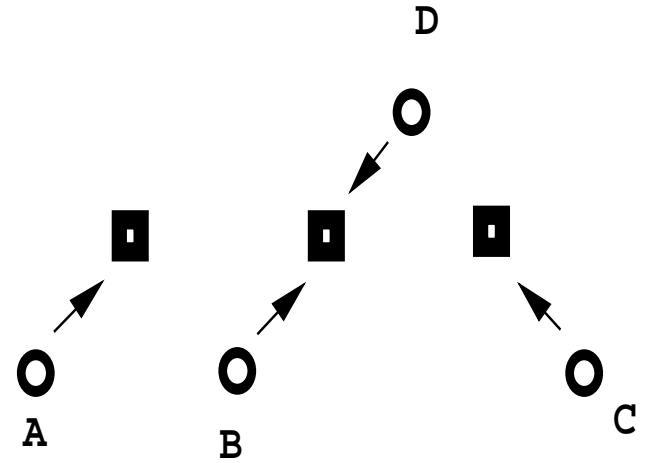

Fig. 1. Non-reciprocal pairwise interactions

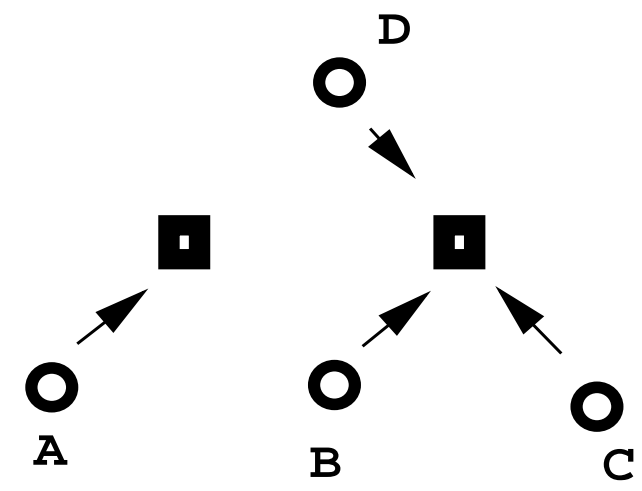

Fig. 2. Non-reciprocal interactions between groups three players

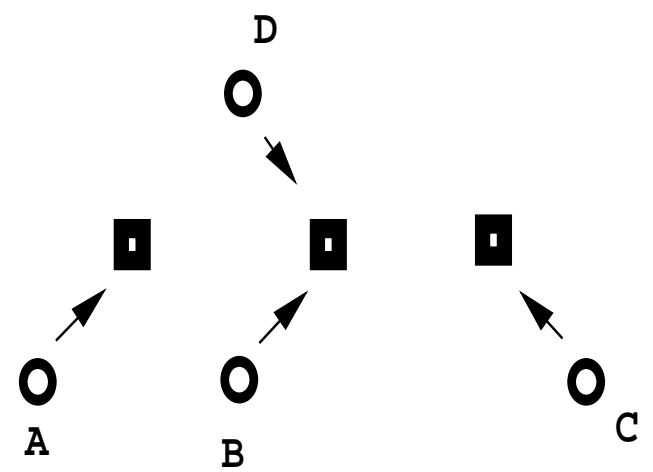

Fig. 3. Interactions between a random number of players

Example 2. In Figure 2 there are four sources and only two destinations. Node A does not cause any interference to the other nodes but suffers interference from nodes B and D. Nodes B, C, D interfere with each other. This is a situation in which each mobile is involved in interference from two other mobiles but again the interference is not reciprocal.

Example 3. In this example the number of interfering nodes is not fixed. A suffers interference from 2 nodes, 
B and D suffer interference from a single other node and

C does not suffer (and does not cause) interference.

All examples exhibit asymmetric realizations and nonreciprocity. We next show how such a situation can still be considered as symmetric (due to the fact that we consider distributions of nodes rather than realizations). Assume that the location of the transmitters follow a Poisson distribution with parameter $\lambda$ over the two dimensional plane. Consider an arbitrary user A. Let $r$ be the interference range. Then the number $N$ of transmitters within the interference range of the receiver of A has a Poisson distribution with parameter $\lambda \pi r^{2} / 2$. Since this holds for any node, the game is considered to be symmetric. The reason that the distribution is taken into account rather than the realization is that we shall assume that the actions of players will be taken before knowing the realization.

\section{B. Model}

We describe in this part notations of our model.

- There is one population of users. The number of users is large.

- We assume that there are pure strategies. Each member of the population chooses from the same set of strategies $\mathcal{A}=\{1,2, \ldots, N\}$.

- Let $M:=\left\{\left(x_{1}, \ldots, x_{N}\right) \mid x_{j} \geq 0, \sum_{j=1}^{N} x_{j}=\right.$ $1\}$ the set of probability distributions over the $N$ strategies. $M$ can be interpreted as the set of mixed strategies. It is also interpreted as the set of distributions of strategies among the population, where $x_{j}$ represents of proportion of users choosing the strategy $j$. A distribution $x$ is sometime called the "state" or "profile" of the game.

- The number of opponents $K$ of a user is a random variable in the finite set $\left\{0,1, \ldots, k_{\max }\right\} . k_{\max }$ is the maximum number of opponents interacting simultaneously with a user. We assume that we can ignore cases of interaction with more than $k_{\max }$ players. This value depends on the node density and the transmission range. When making a choice of a strategy, a player knows the distribution of $K$ but not its realization.

- The payoff of all players functions (identical for each member of the population) of the player's own behavior and opponents' behavior. Each user's payoff depends on opponents' behavior through the distribution of opponents' choices and of their number. The expected payoff of a user playing strategy $j$ when the state of the population is $x$, is given by

$$
f_{j}(x)=\sum_{k=0}^{k_{\max }} \mathbb{P}(K=k) u_{k}(j, x, \ldots, x),
$$

$j=1, \ldots, N$ where $u_{k}$ is the payoff function given that the number of opponents is $k$. Although the payoffs are symmetric, the actual interference or interactions between two players that use the same strategy need not be the same, allowing for nonreciprocal behavior. The reason is that the latter is a property of the random realization whereas the actual payoff already averages over the randomness related to the interactions, the number of interfering players, the topology etc.

\section{Evolutionary Stable Strategies: ESS}

Suppose that, initially, the population profile is $x \in M$. The average payoff is

$$
f(x, x)=\sum_{j=1}^{N} x_{j} f_{j}(x) .
$$

Now suppose that a small group of mutants enters the population playing according to a different profile mut $\in M$. If we call $\epsilon \in(0,1)$ the size of the subpopulation of mutants after normalization, then the population profile after mutation will be $\epsilon m u t+(1-\epsilon) x$. After mutation, the average payoff of non-mutants will be given by $\epsilon f(x$, mut $)+(1-\epsilon) f(x, x)$ where

$$
f(x, m u t)=\sum_{j=1}^{N} x_{j} f_{j}(m u t) .
$$

Analogously, the average payoff of a mutant is

$$
(1-\epsilon) f(\text { mut }, x)+\epsilon f(\text { mut, } m u t) .
$$

Definition 1: A strategy $x \in M$ is an ESS if for any mut $\neq x$, there exists some $\epsilon_{m u t} \in(0,1)$, which may depend on mut, such that for all $\epsilon \in\left(0, \epsilon_{m u t}\right)$

$$
A_{1}>A_{2}
$$

where $A_{1}:=\epsilon f(x, m u t)+(1-\epsilon) f(x, x)$, and $A_{2}:=$ $(1-\epsilon) f($ mut,$x)+\epsilon f($ mut, mut $)$.

That is, $x$ is ESS if, after mutation, non-mutants are more successful than mutants. In other words, mutants cannot invade the population and will eventually get extinct.

Equation (2) may be rewritten as

$$
\begin{array}{r}
\epsilon(-f(\text { mut }, \text { mut })+f(x, \text { mut })) \\
+(1-\epsilon)(f(x, x)-f(\text { mut }, x))>0 .
\end{array}
$$


There is a close relation between ESS and nash equilibrium of the following matrix-game

$$
\Gamma:=\left(\left\{1,2, \ldots, k_{\max }\right\}, \mathcal{A}, r(.)\right)
$$

where $r: \mathcal{A}^{k_{\max }} \rightarrow \mathbb{R}, \quad r\left(a_{1}, a_{2}, \ldots, a_{k_{\max }}\right)=$ $\sum_{l=1}^{k_{\max }} u_{l}\left(a_{1}, a_{2}, \ldots, a_{l}\right) \mathbb{P}(K=l)$.

It is easy to see that inequality (3) is equivalent to the two following conditions:

- Nash equilibrium condition of the matrix-game $\Gamma$.

$$
\forall \text { mut } \in M, f(\text { mut }, x) \leq f(x, x),
$$

- Stability condition

$$
\begin{array}{r}
\text { if } m u t \neq x, \text { and } f(\text { mut }, x)=f(x, x) \\
\text { then } f(\text { mut }, \text { mut })<f(x, \text { mut }) .
\end{array}
$$

\section{Replicator dynamics}

Replicator dynamics is one of the most studied dynamics in evolutionary game theory. The replicator dynamics has been used for describing the evolution of road traffic congestion in which the fitness is determined by the strategies chosen by all drivers [9]. It has also been studied in the context of the association problem in wireless networks in [10]. We introduce the replicator dynamics which describes the evolution in the population of the various strategies. In the replicator dynamics, the share of a strategy $j$ in the population grows at a rate proportional to the difference between the payoff of that strategy and the average payoff of the population. The replicator dynamic equation is given by

$$
\dot{x}_{j}(t)=\mu x_{j}(t)\left[f_{j}(x(t))-\sum_{k=1}^{N} x_{k} f_{k}(x(t))\right] .
$$

where $\mu$ is some positive constant. The parameter $\mu$ can be used to tune the rate of convergence and it may be interpreted as as the rate that a player of the population participates in a (local interaction) game. In biology, it can represent the probability that an animal finds a resource available.

\section{Multiple ACCESS GAME}

The static multiple access game considered here is a generalization of the random access game considered by Inaltekin and Wicker in [3]. Multiple Access Game introduces the problem of medium access. We assume that mobiles are randomly placed over a plane. All mobiles use the same fixed transmission range of $r$. The channel is ideal for transmission and all errors are due to collision. A mobile decides to transmit a packet or not to transmit to a receiver when they are within transmission range of each other. Interference occurs as in the ALOHA protocol: if more than one neighbors of a receiver transmit a packet at the same time then there is a collision. The Multiple Access Game is a nonzero-sum game, the mobiles have to share a common resource, the wireless medium. In this game, the parameter $\mu$ represents the probability that a mobile has its receiver $R(i)$ within its range. When a mobile $i$ transmits to $R(i)$, all mobiles within a circle of radius $r$ centered at $R(i)$ cause interference to the node $i$ for its transmission to $R(i)$. This means that more than one transmission within a distance $r$ of the receiver in the same slot cause a collision and the loss of mobile's $i$ packet at $R(i)$.

Each of the mobiles has two possible strategies: either to transmit $(T)$ or to stay quiet $(S)$. If mobile $i$ transmits a packet, it incurs a transmission cost of $\delta \geq 0$. The packet transmission is successful if the other users don't transmit (stays quiet) in that given time slot, otherwise there is a collision and the corresponding cost is $\Delta \geq 0$. If there is no collision, user $i$ gets a reward of $V$ from the successful packet transmission. We suppose that the reward $V$ is greater than the cost of transmission $\delta$. When all users stay quiet, they have to pay a regret cost $\kappa$. If $\kappa=0$ the game is called degenerate multiple access game. Figure 4 represents an example of interaction of three nodes. The ESS corresponding to any number of nodes $^{1}$ of this game is given in theorem 1 .

node 2

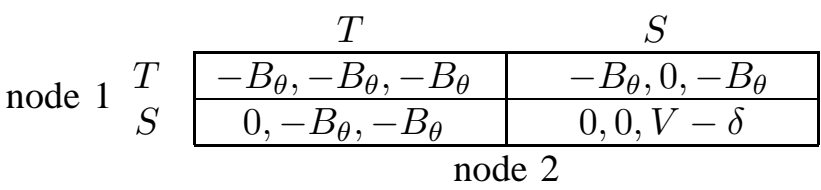

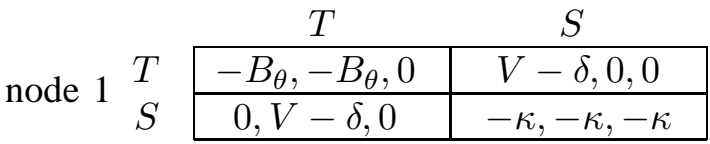

Fig. 4. Multiple access game with three nodes: node chooses a row, node 2 choose a column and node 3 an array with $B_{\theta}=\Delta+\delta$

Let $\mathcal{A}:=\{T, S\}$ be the set of strategies. An equivalent interpretation of strategies is obtained by assuming that individuals choose pure strategies and then the probability distribution represents the fraction of individuals in the population that choose each strategy. We denote by $x$ (resp. $1-x$ ) the population share of strategy $T$ (resp. $S)$.

The payoff obtained by a node with $k$ opponents when it plays $T$ is $u_{k}(T, x)=(-\Delta-\delta)\left(1-\eta_{k}\right)+(V-\delta) \eta_{k}$ where $\eta_{k}:=(1-x)^{k}$, and the node-mutant receives

\footnotetext{
${ }^{1}$ The one-shot game with $n$ nodes has $2^{n}-1$ Nash equilibria and a unique ESS.
} 
$u_{k}(S, x)=-\mu \kappa(1-x)^{k}$ when it stays quiet. The expected payoff of an anonymous transmitter node-mutant is given

$$
\begin{array}{r}
f(T, x):=\mu \sum_{k \geq 0} \mathbb{P}(K=k) u_{k}(T, x) \\
=\mu\left(\begin{array}{c}
\left.-(\Delta+\delta)+(V+\Delta) \sum_{k \geq 0} \mathbb{P}(K=k)(1-x)^{k}\right) \\
=-\mu(\Delta+\delta)+\mu(V+\Delta) G_{K}(1-s) .
\end{array}\right.
\end{array}
$$

where $G_{K}$ is the generating function of $K$. Analogously, we have

$$
\begin{aligned}
f(S, x) & :=\mu \sum_{k \geq 0} \mathbb{P}(K=k) u_{k}(S, x) \\
& =-\mu \kappa \sum_{k \geq 0}(1-x)^{k} \mathbb{P}(K=k) .
\end{aligned}
$$

From equation 1, the expected payoff of any individual in the population where $x$ is the proportion of mobiles which transmit, is given by:

$$
f(x, x)=x f(T, x)+(1-x) f(S, x) .
$$

We next introduce two alternative information scenario that have an impact on the decision making. In the first case, a mobile does not know whether there are zero or more other mobiles in a given local interaction game about to be played. In the second case the mobile has this information, and consequently he transmits with probability one in case no other potential interferers are present. In addition to studying these two cases we shall also consider a third case called the "massively dense" ad-hoc network in which, whenever a mobile participates wishes to transmit, there is at least one other mobile that is involved in the local interaction game.

We denote $\alpha:=\frac{\Delta+\delta}{V+\Delta+\kappa}$, which represents the ratio between the collision cost $-\Delta-\delta$ (cost when there is a collision during a transmission) and the difference between global cost perceived by a mobile $-\Delta-\delta-\kappa$ (collision and regret) and the benefit $V-\delta$ (reward minus transmission cost). When the collision cost $\Delta$ becomes high, the value $\alpha$ converges to one and when the reward or regret cost becomes high, the value $\alpha$ is close to zero.

\section{A. Case 1 : Aloha without sensing}

A transmitter does not know if there are other transmitters at the range of its receiver. Then, even when it is the only transmitter, it has to decide to transmit or not.

Theorem 1: If $\mathbb{P}(K=0)<\frac{\Delta+\delta}{V+\Delta+\kappa}=: \alpha$, then the game has a unique ESS $x_{1}^{*}$ given by

$$
x_{1}^{*}=g^{-1}\left(\frac{\Delta+\delta}{V+\Delta+\kappa}\right)
$$

where $g: x \mapsto \sum_{k=0}^{k_{\max }} \mathbb{P}(K=k)(1-x)^{k}$. a) proof: A mixed equilibrium $x$ is characterized by $f(T, x)=f(S, x)$ i.e

$$
g(x)=\frac{\Delta+\delta}{V+\Delta+\kappa} .
$$

The function $g$ is continuous and strictly decreasing monotone on $(0,1)$ with $g(1)=\mathbb{P}(K=0)$ and $g(0)=1$. Then the equation (11) has a unique solution in $(\mathbb{P}(K=0), 1)$. has, $f(x, m u t)-f($ mut, mut $)=$ $\mu(V+\Delta+\kappa)(s-$ mut $)(g($ mut $)-g(x))$. Thus, $f(x, m u t)-f($ mut, mut) $>0$ (because $g$ is strictly decreasing function) for all mut $\neq x$. This completes the proof.

When a mobile is never alone in his interference area, i.e. $\mathbb{P}(K=0)=0$; the condition $\alpha>0$ is satisfied.

\section{B. Case 2: Aloha with sensing}

A mobile knows when it is the only transmitter at the range of its receiver, and when it is it will thus transmit with probability one. We can say then that the action set is $(T)$ whenever a user has opponents in a local interaction.

Theorem 2: An anonymous user without opponents receives the fitness $f_{0}=V-\delta$. If $\mathbb{P}(K=0)<\frac{\Delta+\delta}{V+\Delta}$, then the game has a unique ESS $x_{2}^{*}$ given by

$$
x_{2}^{*}=g^{-1}\left(\frac{\Delta+\delta+\kappa \mathbb{P}(K=0)}{V+\Delta+\kappa}\right)
$$

where $g: x \mapsto \sum_{k=0}^{k_{\max }} \mathbb{P}(K=k)(1-x)^{k}$.

Proof: The proof is similar as in theorem 1 .

\section{Case 3: Massively dense}

In this case, we take into account only local interactions between users. Then, in this case, mobiles are never alone to transmit during a slot and we have $\sum_{k=1}^{k_{\max }} \mathbb{P}(K=k)=1$.

Theorem 3: The game has always an unique ESS which is solution of the following equation $\sum_{k \geq 1} \mathbb{P}(K=k)(1-x)^{k}=\alpha$.

Proposition 1: The ESSs given in theorems 1,2,3 is asymptotically stable in the replicator dynamics without delays.

b) proof: The replicator dynamics is given by

$$
\dot{x}=(V+\Delta+\kappa) x(1-x)(g(x)-\alpha) .
$$

The function $g$ is decreasing on $(0,1)$ implies that the derivative of the function $x(1-x)(g(x)-\alpha)$ at the ESS is negative. Hence, the ESS is asymptotically stable. 


\section{ESS AND NODES DISTRIBUTION}

In this section, we consider different nodes distributions. We study the existence and the uniqueness of ESS in the different nodes distribution. First, one, we assume that all mobiles have the same number of neighbors $n-1$, i.e., $\mathbb{P}(K=j)=\delta_{n-1}(j)$ and seconde one, we assume that nodes are randomly distributed on a plan following a Poisson point process with density $\lambda$. Due to the page limit, we describe only the poisson distribution. The interested reader is referred to [8] for the dirac distribution.

\section{A. Poisson distribution}

We consider that nodes are distributed over a plan following a Poisson distribution with density $\lambda$. The probability that a node has $i$ neighbors is given by the following distribution.

Cases 1 and 2:

$$
\mathbb{P}(K=k)=\frac{\left(\lambda \pi r^{2}\right)^{k}}{k !} e^{-\lambda \pi r^{2}}, k \geq 0 .
$$

Case 3:

$$
\mathbb{P}(K=k)=\frac{\left(\lambda \pi r^{2}\right)^{k-1}}{(k-1) !} e^{-\lambda \pi r^{2}}, k \geq 1 .
$$

Considering those node distributions and from previous theorems, the unique ESS $x^{*}$ for all cases, is solution of the following equation :

$$
\left\{\begin{array}{cc}
e^{-\lambda \pi r^{2} x_{1}}=\alpha & \text { for case 1 } \\
e^{-\lambda \pi r^{2} x_{2}}=\alpha+\frac{\kappa \mathbb{P}(K=0)}{V+\Delta+\kappa} & \text { for case 2 } \\
\left(1-x_{3}\right) e^{-\lambda \pi r^{2} x_{3}}=\alpha & \text { for case 3 }
\end{array}\right.
$$

Thus we obtain the following equilibria in the different scenario:

$$
\begin{gathered}
x_{1}^{*}=\log \left(\alpha^{-\frac{1}{\lambda \pi r^{2}}}\right), x_{2}^{*}=\log \left(\left(\alpha+\frac{\kappa \mathbb{P}(K=0)}{V+\Delta+\kappa}\right)^{-\frac{1}{\lambda \pi r^{2}}}\right) \\
\operatorname{and} x_{3}^{*}=1-\frac{\text { Lambert } W\left(\lambda \pi r^{2} \alpha e^{\lambda \pi r^{2}}\right)}{\lambda \pi r^{2}},
\end{gathered}
$$

where LambertW $(x)$ is the LambertW function which is the inverse function of $f(w)=w e^{w}$.

\section{OPTIMIZATION ISSUES AND NUMERICAL INVESTIGATION}

We look for the probability of success that can be achieved in a local interaction depending on distribution parameters and also cost parameters. We consider the Poisson distribution with parameters $\lambda$ and $r$.

The probability to have a successful transmission in a local interaction (total throughput) is given by different equation depending on the scenario. In the case 1 we have:

$$
\begin{gathered}
P_{\text {succ }}(\alpha, \lambda)=\mu x_{1}^{*} \sum_{k=0}^{k_{\max }} k \mathbb{P}(K=k)\left(1-x_{1}^{*}\right)^{k} \\
=\mu x_{1}^{*} \sum_{k=0}^{k_{\max }} k \frac{\left(\lambda \pi r^{2}\right)^{k}}{k !}\left(1-x_{1}^{*}\right)^{k} \\
\approx \mu x_{1}^{*}\left(1-x_{1}^{*}\right) \lambda \pi r^{2} \alpha
\end{gathered}
$$

In the case 2 , we have: $P_{\text {succ }}(\bar{\alpha}, \lambda) \approx \mu x_{2}^{*}(1-$ $\left.x_{2}^{*}\right) \lambda \pi r^{2} \bar{\alpha}$.

Proposition 2: The maximum total throughput under poisson distribution is attained when $\alpha=e^{h(\lambda, r)}$ in the case 1 (resp. $\bar{\alpha}=e^{h(\lambda, r)}$ in the case 2) where $h$ is one of the two functions defined by

$$
(\lambda, r) \in \mathbb{R}_{+}^{2} \mapsto \frac{-\left(1+2 \lambda \pi r^{2}\right) \pm \sqrt{1+4\left(\lambda \pi r^{2}\right)^{2}}}{2} .
$$

In the case 3, we have:

$$
\begin{gathered}
P_{\text {succ }}(\alpha, \lambda)=\mu x_{3}^{*} \sum_{k=1}^{k_{\max }} k P(K=k)\left(1-x_{3}^{*}\right)^{k} \\
=\mu x_{3}^{*} \sum_{k=1}^{k_{\max }} k \frac{\left(\lambda \pi r^{2}\right)^{k-1}}{(k-1) !}\left(1-x_{3}^{*}\right)^{k} \\
\approx \mu \alpha x_{3}^{*}\left(1+\lambda \pi r^{2}\left(1-x_{3}^{*}\right)\right) .
\end{gathered}
$$

Proposition 3: There exists a unique $\alpha_{3}^{*}$ in which the total throughput is maximum when $\alpha=\alpha_{3}^{*}$. The $\alpha_{3}^{*}$ is given by

$$
\alpha_{3}^{*}=(1-x) e^{-\lambda \pi r^{2} x}
$$

where $x$ is the unique solution in $[0,1]$ of the following equation :

$$
1+\gamma-x\left(2+5 \gamma+\gamma^{2}\right)+x^{2}\left(4 \gamma+2 \gamma^{2}\right)-\gamma^{2} x^{3}=0
$$

c) proof: The derivative of the function $H:=$ $\frac{\partial P_{s u c c}}{\partial s} \mathrm{~s}$ given by

$H(s)=\left(1+\gamma-s\left(2+5 \gamma+\gamma^{2}\right)+s^{2}\left(4 \gamma+2 \gamma^{2}\right)-s^{3} \gamma^{2}\right) e^{-\gamma s}$.

We prove that the above function is strictly decreasing on $[0,1]$. For that, it is sufficient to study the following function

$G(s)=1+\gamma-s\left(2+5 \gamma+\gamma^{2}\right)+s^{2}\left(4 \gamma+2 \gamma^{2}\right)-s^{3} \gamma^{2}$.

We have $\frac{\partial G(s)}{\partial s}$ is given by

$$
\frac{\partial G(s)}{\partial s}=-\left(2+5 \gamma+\gamma^{2}\right)+2 s\left(4 \gamma+2 \gamma^{2}\right)-3 s^{2} \gamma^{2} .
$$

It is easy to show that the above function is always negative. Since $H(0)=1+\gamma>0$ and $H(1)=-e^{-\gamma}<0$ then the function $H$ is positive for $s \in[0, \bar{s})$ and is negative for $s \in(\bar{s}, 1]$ where $\bar{s}$ is the solution of the equation $G(s)=0$. Since $s^{*}$ is decreasing function on $\alpha$, we conclude that function $P_{\text {succ }}$ is positive if $s \in[0, \bar{s})$ and is negative $s \in(\bar{s}, 1]$. Since the optimal of function $P_{\text {succ }}$ is attained at $\alpha=(1-\bar{s}) e^{-\lambda \pi r^{2} \bar{s}}$ 
The probability of success at the ESS in poisson distribution is represented in figures 5 and 6 . We observe in particular case that when the number of interferes increases, i.e. the rate $\lambda$ in the case of the Poisson distribution, the total throughput increases.

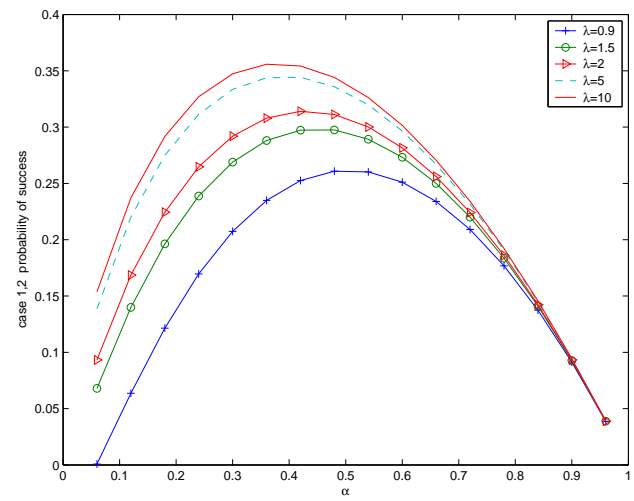

Fig. 5. Probability of success in poisson distribution(cases 1,2).

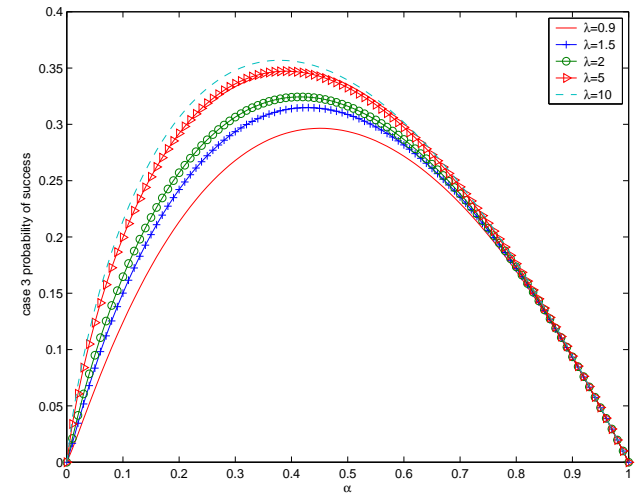

Fig. 6. Probability of success in poisson distribution(case 3).

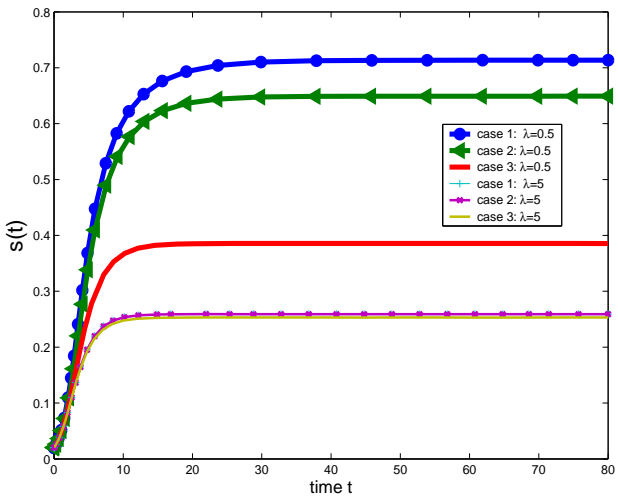

Fig. 7. Evolution of the fraction of transmitters varying the density parameter $\lambda$ (without delays).

In the figures 7 and 8, we describe numerical application of our evolutionary game model with Poisson distribution of nodes under the replication dynamics. We took $n=4=k_{\max }, \Delta=\frac{1}{4}=\delta=\kappa, \lambda=1$ and $V=1$. The initial condition in all these figures is 0.02 . In the figure 7 we compare the evolution of the fraction of transmitters varying the parameter of density $\lambda$ between 0.1 and 5 for the case 1, 2 and 3 respectively. We observe that we have stability for all cases. In figure 8 represents the impact of the parameter $\mu$ on the velocity of the system. We took $\mu$ between 0.1 and 0.5 . without delay. We observe that we have stability but the convergence speed becomes slow when $\mu$ decreases.

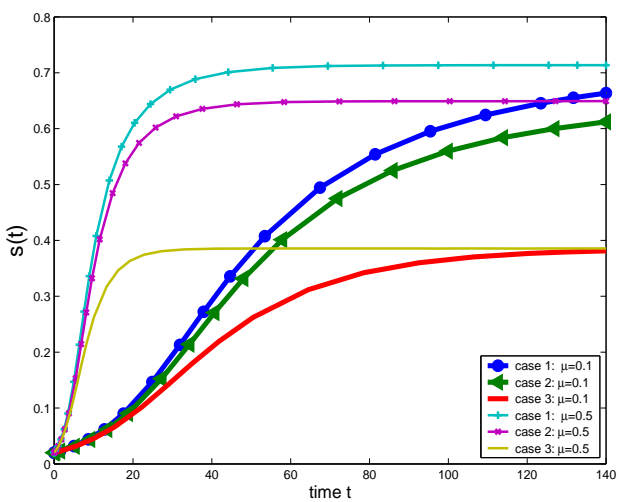

Fig. 8. Impact of the parameter $\mu$ on the velocity of the replicator dynamics without delay.

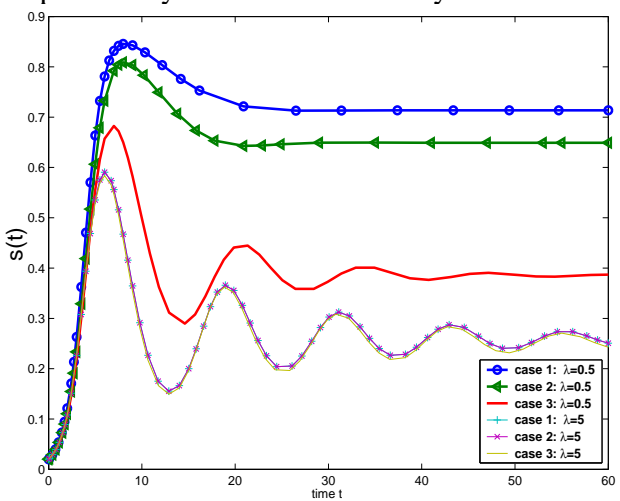

Fig. 9. Evolution of the fraction of transmitters varying the density parameter $\lambda$.

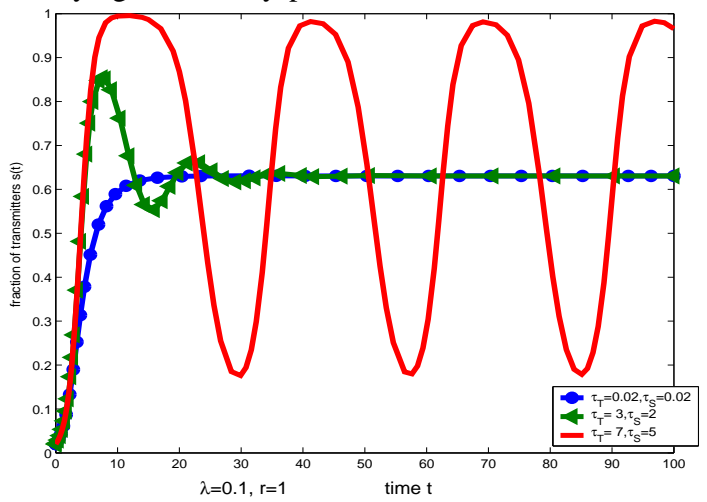

Fig. 10. Impact of the time delay on the stability of the replicator dynamics (case 1).

Now, we study the effect of the time delays on the convergence of replicator dynamics to the evolutionary 
stable strategies in which each pure strategy is associated with its own delay. Let $\tau_{T}$ (resp. $\tau_{S}$ ) be the time delay of the strategy $(T)$ (resp. $(S)$ ). The replicator dynamics becomes

$\dot{x}(t)=\mu x(t)(1-x(t))\left[f\left(T, x\left(t-\tau_{T}\right)\right)-f\left(S, x\left(t-\tau_{S}\right)\right)\right]$

where $f(T, x(t)):=\mu\left(-(\Delta+\delta)+(V+\Delta) e^{-\lambda \pi r^{2} s}\right)$ and $f(S, x(t)):=-\mu \kappa e^{-\lambda \pi r^{2} x}$ in the case 1.In order to study the asymptotically stability of the replicator dynamics (12) around the unique ESS, we linearize (12) at $x^{*}=x_{1}^{*}$. We obtain the following linear delay differential equation

$$
\dot{y}=-c_{1}\left((V+\Delta) y\left(t-\tau_{T}\right)+\kappa y\left(t-\tau_{S}\right)\right)
$$

where $c_{1}:=\mu x^{*}\left(1-x^{*}\right) \alpha\left(1+x^{*}\left(1-x^{*}\right) \lambda \pi r^{2}\right)$, and $y(t)=x(t)-x^{*}$. The following theorem give sufficient conditions of stability of (13) at zero.

Theorem 4 (see [6]): Suppose at least one of the following conditions holds

(i) $(V+\Delta) \tau_{T}+\kappa \tau_{S}<\theta_{a}$,

(ii) $V+\Delta>\kappa$ and $(V+\Delta) \tau_{T}<\frac{(V+\Delta-\kappa) \theta_{a}}{V+\Delta+\kappa}$,

(iii) $V+\Delta<\kappa$ and $\kappa \tau_{S}<\frac{(-V-\Delta+\kappa) \theta_{a}}{V+\Delta+\kappa}$ where

$$
\theta_{a}:=\frac{1}{x^{*}\left(1-x^{*}\right) \mu \alpha\left(1+x^{*}\left(1-x^{*}\right) \lambda \pi r^{2}\right)}
$$

Then the ESS $x^{*}$ is asymptotically stable.

A necessary and sufficient condition of stability of (13) at zero when delays are symmetric is given in theorem 5 .

Theorem 5 (symmetric delay): Suppose that $\tau_{T}=$ $\tau_{S}=\tau$. Then, the ESS $x^{*}$ is asymptotically stable if and only if

$$
\tau<\frac{\pi}{2 x^{*}\left(1-x^{*}\right) \mu \alpha\left(1+x^{*}\left(1-x^{*}\right) \lambda \pi r^{2}\right)(V+\Delta+\kappa)}
$$

The proof uses the following well known lemma (see [5]) and the references therein.

Lemma 1: The trivial solution of the linear delay differential equation

$$
\dot{z}(t)=-a z(t-\tau), \tau, a>0
$$

is asymptotically stable if and only if $2 a \tau<\pi$.

The fraction of transmitters in the population is represented in figure 10 for $\lambda=0.5$ and $r=1$. The delays $\tau_{T}$ and $\tau_{S}$ are between 0.02 and 7 . The system is stable for $\tau_{T}=\tau_{S}=0.02$ or $\tau_{T}=3, \tau_{S}=2$. For $\tau_{T}=7$ and $\tau_{S}=5$ the system is unstable. We display an oscillatory behavior of the population as function of time. The trajectory are seen to converge to periodic ones. All turn out to confirm the stability condition that we obtained in theorem 4 . In the figure 9 we compare evolution of the fraction of transmitters varying the parameter of density $\lambda$ between 0.1 and 5 for the case 1, 2 and 3 respectively. In this figure, the time delays are respectively 3 and 2 . Note that in this figure the equilibrium point is decreasing function in the density parameter $\lambda$. Indeed, when the density of nodes increases, the number of mobiles share a receiver increases. To avoid collision, the nodes decrease the probability of transmission. We observe also that for $\lambda=5$, we have stability but the convergence speed is slow than for $\lambda=0.1$.

\section{CONCLUSION}

In this paper we have adapted the theory of evolutionary games with a random number of players. This adaptation is needed in order to apply this theory for the study of access game and particularly in wireless networks. We have proposed different scenario based on the level of information for each player. In all cases, we have obtained the existence and uniqueness of the ESS, we have proposed optimization issues for the transmission probability of success and finally, we have studied the impact of delay in the convergence to the ESS of the replicator dynamics.

\section{ACKNOWLEDGMENTS}

We thank two anonymous reviewers for their many suggestions for improving this paper.

\section{REFERENCES}

[1] N. Bonneau, E. Altman, M. Debbah, and G. Caire. An Evolutionary Game Perspective to ALOHA with Power Control. 19th International Teletraffic Congress, Beijing, China, Aug. 29-Sep.,2005.

[2] J. Hofbauer and K. Sigmund. Evolutionary Games and Population Dynamics. Cambridge University Press, Cambridge, UK, 1998.

[3] H. Inaltekin and S. Wicker, A One-shot Random Access Game, Inter. Conference on Wireless Networks, Comm. and Mobile Computing, 2005.

[4] J Maynard Smith and GR Price. The logic of animal conflict, Nature 246,15-18,1973.

[5] H. Tembine, E. Altman and R. El-Azouzi. Delayed Evolutionary Game Dynamics applied to the Medium Access Control, Bionetworks 2007, Pisa, Italy.

[6] H. Tembine, E. Altman and R. ElAzouzi. Asymmetric Delay in Evolutionary Games, Valuetools, 2007.

[7] H. Tembine, E. Altman, R. El-Azouzi and Y. Hayel. Multiple Access Game in Ad-hoc Network, GameComm 2007.

[8] H. Tembine, E. Altman, R. El-Azouzi and Y. Hayel. Evolutionary games with random number of interacting players applied to access control, LIA technical report, 2007.

[9] W.H. Sandholm. Potential games with continuous player sets. J. Eco. Theory, 97:81-108, 2001.

[10] Shakkottai S., Altman E., and Kumar A. The case for noncooperative multihoming of users to access points in ieee 802.11 wlans. IEEE Infocom, 2006.

[11] J.W. Weibull. Evolutionary Game Theory. Cambridge, MA: MIT Press, 1995. 